\title{
Review Article \\ Does Smoking Act as a Friend or Enemy of Blood Pressure? Let Release Pandora's Box
}

\begin{abstract}
Aurelio Leone
Department of Internal Medicine, City Hospital Massa, Via Provinciale 27, 19030 Castelnuovo Magra SP, Italy

Correspondence should be addressed to Aurelio Leone, reliol@libero.it

Received 21 June 2010; Accepted 3 January 2011

Academic Editor: Undurti N. Das

Copyright () 2011 Aurelio Leone. This is an open access article distributed under the Creative Commons Attribution License, which permits unrestricted use, distribution, and reproduction in any medium, provided the original work is properly cited.

In spite of the great number of observations which show the certainty of cardiovascular damage from smoking, the opinions on that are not yet unanimous. There is a discrepancy that could be attributed to the lack of reproducible data particularly in some epidemiological studies. On the contrary, experimental findings conducted on both animals and humans give evidence of exactly reproducible results of cardiovascular alterations and among these the course of Blood Pressure (BP). Findings identify an increase in BP of active smokers or non-smokers exposed to passive smoking, while a lot of others refer a lowering of BP due to smoking. This discrepancy could be explained as follows. Initially, a vasoconstriction mediated by nicotine causes acute but transient increase in systolic BP. This phase is followed by a decrease in BP as a consequence of depressant effects played chronically by nicotine itself. Simultaneously, carbon monoxide is acting directly on the arterial wall causing, in the long run, structurally irreversible alterations. At this time, there is a change in BP that increases again, and often constantly, its levels following chronic exposure. Changes in response to antihypertensive drugs have been observed in hypertensive smokers since smoking influences metabolic steps of the drugs.
\end{abstract}

\section{Introduction}

Tobacco smoke is a term indicating cigarette smoking, cigar smoking, and pipe smoking. Usually, the main reports concerning the relationship between smoking and cardiovascular alterations are attributed to cigarette smoking since systematic studies on the harm caused by pipe and cigars are yet lacking.

There are a lot of reports that identify cardiovascular system as one of the major target organs for smoking [112]. Either active or passive exposure to smoking causes damage to the heart and blood vessels although pathological mechanisms of damage may differ with regards to the type of action but not for that is concerning chemical toxics responsible of the alterations [13-30].

In spite of the great number of observations which show the certainty of cardiovascular damage from smoking, the opinions are not yet unanimous. There is a discrepancy that could be attributed to the lack of reproducible data particularly in some epidemiological studies. On the contrary, experimental findings conducted on both animals and humans give evidence of exactly reproducible results of cardiovascular alterations.

Adverse effects on the heart and vessels are mediated by many chemical compounds that are usually concentrated and condensed into tobacco mixtures [23]. Over 4000 chemicals have been identified in smoke, and a large majority of these have carcinogenic and/or negative cardiovascular effects in humans and animals. Chemical compounds of smoking cause both structural and functional alterations of heart and blood vessels, although with different results which are depending on several factors related to the type of smoking, environment, and subject exposed.

Worldwide, more than 3 million people currently die each year from smoking, half of them before the age of 70 , an enormous human cost, and more than one and third have cardiovascular events that often determine permanent disability of affected subjects $[24,25]$. There are more than 1 billion smokers in the world with an increased/decreased/again increased smoking habit.

Main cardiovascular diseases related to cigarette smoking are listed in Table 1. 
TABLE 1: Main cardiovascular diseases related to cigarette smoking.

\begin{tabular}{l}
\hline Coronary artery disease \\
Stroke and cerebrovascular disease \\
Peripheral artery disease \\
Aortic aneurysm \\
Hypertension \\
Heart failure \\
Arrhythmias \\
Endothelial dysfunction \\
Atherosclerosis \\
\hline
\end{tabular}

Among cardiovascular parameters, blood pressure (BP) is adversely influenced by tobacco smoke with a high rate by a mechanism yet under discussion. In addition, it is not clear if smoking exposure causes a rise or reduction of blood pressure and, otherwise, also if the occurrence of hypertension in smokers is a consequence of the greatest number of hypertensive people independently from smoking, or smoking actively contributes to changes in BP.

The purpose of this paper is to discuss those results that have been reached by the analysis on the relationship between smoking and BP in both smokers and nonsmokers who were passively exposed. The possible interference of smoking on the effects of the most used antihypertensive drugs is also treated.

\section{Blood Pressure in Active Smokers}

Active smokers can display BP values which vary widely according to a great number of individual, racial, and lifestyle factors. Moreover, changes in BP have been documented in the same smoker while he is smoking a cigarette or not. While a smoker is actively smoking, transiently sympathetic responses, which acutely raise BP levels, usually occur.

Reports emphasize that hypertension or hypotension can be associated with cigarette smoking in active smokers but there is no evidence on the BP measures whether smoking was lacking.

Some findings $[31,32]$ identified that cigarette smoking in males was inversely related to systolic BP with a reduction of $1.3 \mathrm{mmHg}$ in $1.1 \%$ of light smokers, $3.8 \mathrm{mmHg}$ in $3.1 \%$ of moderate smokers, and $4.6 \mathrm{mmHg}$ in $3.7 \%$ of heavy smokers when these individuals were compared to nonsmokers. There was no clear relation with diastolic blood pressure. This finding was conducted in an oriental population enrolled in the study, but also in Western countries blood pressure reduction was observed primarily in young smokers [32].

In addition, epidemiologic surveys [33-41], although not all, demonstrated that individuals who smoked a different number of cigarettes had lower blood pressure than that of non-smokers. Such a characteristic occurred in males, females, adolescents, adults, and different races. However, this observation was attributed primarily to chronic smoking. Associated loss in body weight of active smokers contributes to lowering BP.
TABLE 2: Cardiovascular parameters particularly involved in smokers.

\begin{tabular}{l}
\hline Systolic BP \\
Heart rate \\
Endothelium-dependent vasodilation \\
\hline
\end{tabular}

Such data contrast strongly with the results obtained in active smokers while they are smoking a cigarette as well as in dated chronic smokers [32, 42-45].

These individuals display an evident increase in blood pressure that seems to be clearly related to the toxic effects of nicotine and carbon monoxide of acute type but, particularly for that concerns carbon monoxide, also of chronic type with structural arterial lesions associated. Structural alterations, in the run, tend to change the behaviour of BP that becomes irreversibly elevated although it was starting from increased levels initially responsive to smoking cessation.

Nowadays, there is evidence that changes in vascular wall begin as early as a smoker begins with smoking but they are of no estimation because of masked damage, as that will be described ahead.

\section{Blood Pressure in Passive Smokers}

Passive smokers display different levels of BP depending on the type and duration of exposure to environmental tobacco smoke.

Increased levels of BP, particularly systolic BP, usually follow acute but transient exposure [46]. Occasionally, there is evidence of hypotension followed, however, by stable hypertension in those individuals exposed for long time to passive smoking even if exposure occurs irregularly.

Some concepts are worthy to be clarified to better understand this occurrence.

An obvious consideration is that acute but transient exposure to passive smoking of a non-smoker individual makes him susceptible of main smoking compounds, primarily nicotine but also carbon monoxide, which have, initially, hypertensive effects directly or indirectly throughout adrenergic and sympathetic stimulation on arterial bed. Similarly, increased heart rate can, usually, be identified. Prolonging the exposure, these parameters $[1-3,5,17,18]$ meet some changes which depend on a large number of factors related to cardiovascular parameters. They influence differently BP levels at the end of isolated acute exposure. Physiology, biochemical characteristics and lifestyle interfere with BP in exposed individuals. Table 2 shows the main cardiovascular parameters involved.

Baseline levels usually tend to be reached after the exposure in a variable but short time and adrenergic and sympathetic profile of the individual also contributes to that.

Finally, lifestyle is a strong positive or adverse factor to restore cardiovascular parameters, particularly systolic blood pressure, according to respectively regular physical exercise performed by the individual or lacking that. 
BP is a clinical parameter of easy assessment and often linked to endothelial dysfunction. Such a statement is particularly true for the essential hypertension [47, 48]. Moreover, smoking and endothelial dysfunction are closely related in passive smokers [19].

The acute response of $\mathrm{BP}$ to environmental tobacco smoke would seem to determine an increase in systolic BP levels in some reports $[49,50]$, whereas others [51] did not conclude for this statement.

The possible hypothesis by which smoking compounds influence BP could be explained as follows. Initially, a vasoconstriction mechanism mediated by nicotine causes acute but transient increase in systolic BP. This phase is followed by a decrease in $\mathrm{BP}$ as a consequence of depressant effects played chronically by nicotine. Simultaneously, carbon monoxide is acting directly on the arterial wall causing, in the long run, structurally irreversible alterations. At this time, there is a change in BP that increases again, and often constantly, its levels [29]. Such a hypothesis explains BP changes following chronic exposure. On the contrary, acute exposure to passive smoking determines a transient increase in systolic BP due to a combined effect of nicotine that acts by endothelial dysfunction and sympathetic stimulation, and carbon monoxide which exerts its toxic effects directly [52-55]. Increased systolic BP after acute exposure to passive smoking was found also by Mahmud and Feely [56], whereas Leone and Corsini [57] documented a decrease in BP following repeated acute exposure to passive smoking. Decrease in $\mathrm{BP}$ was proportional to the increase in carboxyhemoglobin concentrations. Diastolic BP would seem to be affected weakly by environmental tobacco smoke exposure.

These observations identify no uniform course of blood pressure in both active smokers and non-smokers exposed and that concept needs to be clarified by the hypothesis of masked cardiovascular damage.

\section{Masked Cardiovascular Damage}

The phenomenon of masked hypertension from smoking was, firstly, described by Leone et al. [32] as an explanatory hypothesis of why no unanimous opinion existed on the relationship between cigarette smoking and BP. In the time, that phenomenon has found scientific support.

From up to here discussed data, a significant observation emerges: a different response characterizes BP in actively or passively exposed smokers due to the fact that the parameter is assessed immediately after an acute exposure to environmental tobacco smoke or after a chronic and prolonged exposure. Acute exposure is followed by a transient but significant increase in systolic BP, whereas chronic exposure may be followed by reduced or increased BP depending on the presence of reversible or irreversible alterations of the arterial wall caused by smoking compounds, particularly carbon monoxide. These alterations are, for a variable time, masked by the paralyzing action exerted by nicotine on ganglionic ends that follows initial stimulation.

Acute exposure to passive smoking influences adversely either blood vessel dilation since there is a reduced release of nitric oxide, or arterial stiffness. Consequently, an increase in BP $[33,45,47,48]$ is observed. These changes on arterial stiffness, and then, BP usually occur before they are clinically manifested [16] and are greater than those seen when a smoker smokes a single cigarette. Although this type of changes affecting BP is, usually, proven lately, there is evidence, however, that it begins acutely while an individual smokes [14].

In conclusion, even if assessing systolic BP immediately after environmental smoking exposure may be difficult unless in experimental findings, one cannot deny its increase and, consequently, its interpretation as a marker of smoking exposure.

As already described, nicotine may mask the effects of carbon monoxide on arterial wall for a long time. Adverse effects, usually, will appear when they will be of structurally severe degree so that to induce stable hypertension.

\section{Antihypertensive Drugs}

A large number of smoker individuals, primarily aged heavy smokers, use antihypertensive drugs to fight hypertension similarly to that occurs in hypertensive non-smokers.

Often some of these drugs meet a change in their mechanism of action because of a close interaction with the main compounds of tobacco smoke, particularly nicotine and its metabolites.

Of the main classes of antihypertensive drugs (Table 3 ), beta-blockers feel primarily the adverse effect of smoking since smoking compounds influence adversely the action and efficacy of beta-blocker drugs through a complex number of effects that involve metabolic response of adrenergic and sympathetic system [58-60].

Beta-blockers have been shown to be less effective to fight elevated BP and heart rate in habitual smokers compared with non-smokers in two large-scale epidemiological surveys $[61,62]$.

Metabolic changes in response to propranolol infusion have been observed particularly in elderly since physiologically autonomic nervous system meets an impairment of different degree and is, also, adversely influenced by exposure to smoking in aged people [63]. In addition, there is evidence that the sensitivity of baroreceptor reflex in elderly is impaired with a decrease in its function [64, 65]. The decrease in sensitivity of baroreceptors is usually interpreted as a consequence of increased aortic stiffness.

A study analyzed the effect of aging on metabolic steps of beta-adrenergic system [66]. It demonstrated that isoproterenol that is able to increase significantly heart rate and BP needed higher concentrations to raise heart rate of 25 beats per minute and the dose of propranolol that reduced usually heart rate response was poorly effective. This effect would seem to be related to a metabolic change in the reactivity of catecholamine receptors [67].

More recent findings [68] have shown that third generation beta-1-adrenoceptor antagonists acting by an endothelium-dependent vasodilatory mechanism, like nebivolol, can reduce the adverse effects of smoking and have a positive 
TABLE 3: Common classes of antihypertensive drugs and their response to smoking.

\begin{tabular}{lll}
\hline Drug & Mechanism of action & Response to smoking \\
\hline Beta-blockers & Inhibition beta 1 receptors & Highly reduced $(+++)$ \\
ACE-Inhibitors & Blocked conversion Angiotensin 1 to Angiotensin II & Highly reduced $(+++)$ \\
Calcium Antagonists & Block entry of calcium into vascular smooth cells & Reduced $(++/-)$ \\
Diuretics & Decreased body sodium and extracellular fluid volume & Highly reduced $(+++)$ \\
Angiotensin receptor blockers & Block AT1 receptor & not yet known $(--/+$ ?) \\
\hline
\end{tabular}

+++ : strongest reduction

$++/-$ : moderate reduction

$+:$ mild reduction

$--1+$ : increase

? : not yet established.

action on endothelial function that was, however, limited only to light smokers.

ACE-inhibitors are drugs largely used to reduce hypertension since they interfere with the conversion of angiotensin I to artery-constricting angiotensin II, the strongest known vasoconstrictor. Blocking the production of angiotensin II results in arterial vasodilation followed by reduction in BP. ACE-inhibitors currently are recommended as first-line therapy for hypertension in certain patient populations, primarily diabetic individuals, because of their safety and efficacy.

There is evidence that cigarette smoking reduces dramatically the benefits of ACE inhibitors in treated hypertensive people. Moreover, the response to ACE inhibitors of some groups of individuals with hypertension complicated primarily by diabetic renal disease is as strongly impaired as worsening of those symptoms which are usually improved by using these drugs [69]. The onset of microalbuminuria in diabetic patients would be related to smoking and hyperglycemia.

Calcium antagonists are a class of antihypertensive drugs that interfere with calcium metabolism at different levels, primarily calcium channels. They are currently used for treating hypertension.

Usually, calcium deposits are found into the arterial wall with an increase in their content associated particularly with aging. Since cigarette smoking induces vasoconstriction and a major incidence of thrombi formation where calcium plays a strong metabolic role, there is evidence that hypertensive smokers could be usefully treated by vasodilators drugs which antagonize calcium deposit [70]. Therefore, using calcium antagonists in hypertensive smokers could be a rationale intervention even if these drugs also are adversely influenced by cigarette smoking. Preventive effects against the damage from smoking would be identified by using calcium antagonists together with nitroglycerin in coronary artery disease [70].

A strongly adverse metabolic relationship exists between smoking and diuretics.

Diuretics are a complex class of drugs largely used for the treatment of hypertension $[71,72]$. With the exception of the antagonists of aldosterone, the main mechanism of action of these drugs consists of inhibiting ion transporters in the luminal membrane of the renal tubule. There is evidence that most of the data concerning the hypotensive action of diuretics may be explained by the analysis of thiazide-type diuretic action. These drugs, which are used in the treatment of hypertension with a major rate than that of other diuretics and often in combination, act by sodium depletion or by a direct vascular effect independent of natriuresis [73].

Smoking influences strongly diuretic treatment of hypertensive smokers since it exerts an adverse effect on BP lowering primarily due to nicotine action. It is known by long time that either intravenous injection of nicotine or smoking one or two cigarettes has similar antidiuretic action in men [74].

Finally, little is known about the relationship between Angiotensin receptor blockers and smoking. Indeed, largescale findings on this matter are yet lacking.

An experimental study [75] suggests a positive effect of Valsartan, an Angiotensin receptor blocker, on the alterations caused by smoking on vascular endothelium. The drug prevented smoking from impairing acetylcholine vasodilation. There was evidence that acute single-cigarette smoking caused a dysfunction of endothelium-dependent, but not endothelium-independent, vasodilation of rat cerebral vessel in vivo. The effect was not mimicked by intravenous nicotine. Therefore, Angiotensin 1 receptor blockade could prevent smoking-induced impairment of endothelium-dependent vasodilation although large-scale findings are yet missing and experimental data should be obtained.

Described data undoubtedly demonstrate that all the major classes of drugs commonly used for the treatment of hypertension are adversely influenced by smoking and, consequently, the goal of an effective treatment could fail in hypertensive smokers independently by the mechanism of action or choice of antihypertensive drug.

\section{Conclusion}

Two basic concepts arise from the analysis of exposed data. They match Pandora's box of Greek mythology [76].

Pandora's jar is a box where all existing evils are contained inside, when it is closed by a lid, to do not harm individuals. Therefore, there is an apparently good health. On the contrary, when the lid is removed all evils come out damaging heavily humans. 
The first concept to stress is the different response of BP to smoking compounds that depends on the type of smoking, its duration, and onset of BP increase.

Initially, young smokers usually display normal or even low values of BP with no evident signs of vascular damage that, however, are beginning although not well evident clinically. Therefore, it would seem an illogical suggestion to forbid smoking to these individuals who are, apparently, in good health. One can consider this stage as that of closed Pandora's box which masks the damage contained inside the box. This could be also identified as the stage where tobacco smoke acts as an apparent friend of BP because of normal or lower levels.

The phenomenon of masked hypertension by either active or passive smoking well correlates the duration of smoking exposure and onset of BP increase. A growing literature highlights the role of this occurrence $[77,78]$. The evidence now suggests that to improve BP, we require to forbid absolutely smoking as early as it has begun. Indeed, masked hypertension seems to be associated particularly with passive smoking in a dose-related manner, and low physical activity, increased heart rate, and postural haemodynamic reaction, all effects similar to those of sympathetic stimulation, may be potential accelerators of that phenomenon. Therefore, masked hypertension causes a type of damage that, pathologically, starts low and goes slow, but in progress, for many years even in case of pharmacological treatment.

When vascular wall alterations are evident and, more often, irreversible, open Pandora's box releases all unmanageable effects which become the structural substrate for hypertensive alterations. This stage represents tobacco smoke that is acting as enemy of BP.

Secondly, when there is evidence of hypertension to be treated, the response to antihypertensive drugs in smokers is usually impaired since biochemical and metabolic interference exists between cigarette smoking and antihypertensive drugs, although some classes of antihypertensive drugs would less the effects of smoking.

\section{References}

[1] A. Leone, "Cardiovascular damage from smoking: a fact or belief?” International Journal of Cardiology, vol. 38, no. 2, pp. 113-117, 1993.

[2] C. B. Sherman, "Health effects of cigarette smoking," Clinics in Chest Medicine, vol. 12, no. 4, pp. 643-658, 1991.

[3] A. Leone, "The heart: a target organ for cigarette smoking," Journal of Smoking-Related Disorders, vol. 3, pp. 197-201, 1992.

[4] US Department of Health, Education, and Welfare, "The health consequences of smoking: a report of the Surgeon General. Cardiovascular disease," DHHS Publication no. (PHS) 84-50204, US Department of Health and Human Services, Public Health Service Office of Smoking and Health, Rockville, Md, USA, 1983.

[5] A. Leone, "Cigarette smoking and health of the heart," Journal of the Royal Society of Health, vol. 115, no. 6, pp. 354-355, 1995.

[6] E. C. Hammond and L. Garfinkel, "Coronary heart disease, roke, and aortic aneurysm. Factors in the etiology," Archives of Environmental Health, vol. 19, no. 2, pp. 167-182, 1969.
[7] P. E. McBride, "The health consequences of smoking: cardiovascular diseases," Medical Clinics of North America, vol. 76, no. 2, pp. 333-353, 1992.

[8] W. S. Aronow, "Effect of passive smoking on angina pectoris," New England Journal of Medicine, vol. 299, no. 1, pp. 21-24, 1978.

[9] D. Sparrow, T. R. Dawber, and T. Colton, "The influence of cigarette smoking on prognosis after a first myocardial infarction," Journal of Chronic Diseases, vol. 31, no. 6-7, pp. 425-432, 1978.

[10] A. Leone, F. Bertanelli, L. Mori, P. Fabiano, and G. Bertoncini, "Ventricular arrhythmias by passive smoking in patients with pre-existing myocardial infarction," Journal of the American College of Cardiology, vol. 3, p. 256, 1992.

[11] C. Wilhelmsson, J. A. Vedin, and D. Elmfeldt, "Smoking and myocardial infarction," Lancet, vol. I, no. 7904, pp. 415-419, 1975.

[12] O. Auerbach, H. W. Carter, L. Garfinkel, and E. C. Hammond, "Cigarette smoking and coronary artery disease. A macroscopic and microscopic study," Chest, vol. 70, no. 6, pp. 697705, 1976.

[13] D. D. Reid, P. J. S. Hamilton, and P. McCartney, "Smoking and other risk factors for coronary heart disease in British civil servants," Lancet, vol. 2, no. 7993, pp. 979-983, 1976.

[14] S. A. Glantz and W. W. Parmley, "Passive smoking and heart disease: mechanisms and risk," Journal of the American Medical Association, vol. 273, no. 13, pp. 1047-1053, 1995.

[15] A. J. Wells, "Passive smoking as a cause of heart disease," Journal of the American College of Cardiology, vol. 24, no. 2, pp. 546-554, 1994.

[16] C. L. Meinert, S. Forman, D. R. Jacobs, and J. Stamler, "Cigarette smoking as a risk factor in men with a prior history of myocardial infarction. The coronary drug project research group," Journal of Chronic Diseases, vol. 32, no. 6, pp. 415-425, 1979.

[17] S. A. Glantz and W. W. Parmley, "Passive smoking and heart disease. Epidemiology, physiology, and biochemistry," Circulation, vol. 83, no. 1, pp. 1-12, 1991.

[18] L. Baer and I. Radichevich, "Cigarette smoking in hypertensive patients. Blood pressure and endocrine responses," American Journal of Medicine, vol. 78, no. 4, pp. 564-568, 1985.

[19] D. S. Celermajer, M. R. Adams, P. Clarkson et al., "Passive smoking and impaired endothelium-dependent arterial dilatation in healthy young adults," New England Journal of Medicine, vol. 334, no. 3, pp. 150-154, 1996.

[20] A. Leone and M. Lopez, "Oral contraception, ovarian disorders and tobacco in myocardial infarction of woman," Pathologica, vol. 78, no. 1054, pp. 237-242, 1986.

[21] S. Pojola, P. Siltanen, and M. Romo, "Five-year survival of 728 patients after myocardial infarction. A community study," British Heart Journal, vol. 43, no. 2, pp. 176-183, 1980.

[22] A. Leone, "Relationship between cigarette smoking and other coronary risk factors in atherosclerosis: risk of cardiovascular disease and preventive measures," Current Pharmaceutical Design, vol. 9, no. 29, pp. 2417-2423, 2003.

[23] J. C. Byrd, "Environmental tobacco smoke: medical and legal issues," Medical Clinics of North America, vol. 76, no. 2, pp. 377-398, 1992.

[24] R. Peto, A. D. Lopez, J. Boreham, M. Thun, and C. Heath, Mortality from Smoking in Developed Countries: 1950-2000, Oxford University Press, Oxford, UK, 1994.

[25] N. J. Wald and A. K. Hackshaw, "Cigarette smoking: an epidemiological overview," British Medical Bulletin, vol. 52, no. 1, pp. 3-11, 1996. 
[26] A. Leone, F. Bertanelli, L. Mori, P. Fabiano, and A. Battaglia, "Features of ischaemic cardiac pathology resulting from cigarette smoking," Journal of Smoking-Related Disorders, vol. 5, no. 2, pp. 109-114, 1994.

[27] Health or Smoking. Follow-Up Report of the Royal College of Physicians, Pitman Publishing, London, UK, 1983.

[28] P. Astrup, "The arterial wall in atherogenesis," in Atherogenesis, Cavallero, Ed., pp. 77-92, Piccin Medical Books, Padua, Italy, 1965.

[29] A. Leone, "Biochemical markers of cardiovascular damage from tobacco smoke," Current Pharmaceutical Design, vol. 11, no. 17, pp. 2199-2208, 2005.

[30] A. Leone, L. Landini, O. Biadi, and A. Balbarini, "Smoking and cardiovascular system: cellular features of the damage," Current Pharmaceutical Design, vol. 14, no. 18, pp. 1771-1777, 2008.

[31] K. Hughes, W. P. Leong, S. P. Sothy, K. C. Lun, and P. P. B. Yeo, "Relationships between cigarette smoking, blood pressure and serum lipids in the Singapore general population," International Journal of Epidemiology, vol. 22, no. 4, pp. 637643, 1993.

[32] A. Leone, M. Lopez, and G. Picerno, "Role of smoking in coronary heart disease: hypothesis on the possible mechanism of myocardial damage," Minerva Cardioangiologica, vol. 32, no. 7-8, pp. 435-439, 1984.

[33] T. Gordon and W. B. Kannel, "Multiple risk functions for predicting coronary heart disease: the concept, accuracy, and application," American Heart Journal, vol. 103, no. 6, pp. 10311039, 1982.

[34] M. Karvonen, E. Orma, A. Keys, F. Fidanza, and J. Brozek, "Cigarette smoking, serum-cholesterol, blood-pressure, and body fatness observations in Finland," The Lancet, vol. 273, no. 7071, pp. 492-494, 1959.

[35] D. Ballantyne, B. L. Devine, and R. Fife, "Interrelation of age, obesity, cigarette smoking, and blood pressure in hypertensive patients," British Medical Journal, vol. 1, no. 6117, pp. 880-881, 1978.

[36] M. W. Higgins and M. Kjelsberg, "Characteristics of smokers and nonsmokers in Tecumseh, Michigan. II. The distribution of selected physical measurements and physiologic variables and the prevalence of certain diseases in smokers and nonsmokers," American Journal of Epidemiology, vol. 86, no. 1, pp. 60-77, 1967.

[37] C. C. Seltzer, "Effect of smoking on blood pressure," American Heart Journal, vol. 87, no. 5, pp. 558-564, 1974.

[38] P. D. Arkwright, L. J. Beilin, and I. Rouse, "Effects of alcohol use and other aspects of lifestyle on blood pressure levels and prevalence of hypertension in a working population," Circulation, vol. 66, no. 1, pp. 60-66, 1982.

[39] U. Goldbourt and J. H. Medalie, "Characteristics of smokers, non smokers and ex smokers among 10,000 adult males in Israel. II. Physiologic, biochemical and genetic characteristics," American Journal of Epidemiology, vol. 105, no. 1, pp. 75-86, 1977.

[40] J. A. Morrison, K. Kelly, and M. Mellies, "Cigarette smoking, alcohol intake, and oral contraceptives: relationships to lipids and lipoproteins in adolescent school-children," Metabolism, vol. 28, no. 11, pp. 1166-1170, 1979.

[41] M. S. Green, E. Jucha, and Y. Luz, "Blood pressure in smokers and nonsmokers: epidemiologic findings," American Heart Journal, vol. 111, no. 5, pp. 932-940, 1986.

[42] C. Su, "Actions of nicotine and smoking on circulation," Pharmacology and Therapeutics, vol. 17, no. 1, pp. 129-141, 1982.
[43] J. Trap-Jensen, "Effects of smoking on the heart and peripheral circulation," American Heart Journal, vol. 115, no. 1, pp. 263$267,1988$.

[44] P. Hill and E. L. Wynder, "Smoking and cardiovascular disease. Effect of nicotine on the serum epinephrine and corticoids," American Heart Journal, vol. 87, no. 4, pp. 491-496, 1974.

[45] G. U. Cellina, A. J. Honour, and W. A. Littler, "Direct arterial pressure, heart rate, and electrocardiogram during cigarette smoking in unrestricted patients," American Heart Journal, vol. 89, no. 1, pp. 18-25, 1975.

[46] A. Leone, D. Giannini, C. Bellotto, and A. Balbarini, "Passive smoking and coronary heart disease," Current Vascular Pharmacology, vol. 2, no. 2, pp. 175-182, 2004.

[47] J. A. Panza, A. A. Quyyumi, J. E. Brush, and S. E. Epstein, "Abnormal endothelium-dependent vascular relaxation in patients with essential hypertension," New England Journal of Medicine, vol. 323, no. 1, pp. 22-27, 1990.

[48] S. John and R. E. Schmieder, "Impaired endothelial function in arterial hypertension and hypercholesterolemia: potential mechanisms and differences," Journal of Hypertension, vol. 18, no. 4, pp. 363-374, 2000.

[49] O. T. Raitakari, M. R. Adams, R. J. McCredie, K. A. Griffiths, and D. S. Celermajer, "Arterial endothelial dysfunction related to passive smoking is potentially reversible in healthy young adults," Annals of Internal Medicine, vol. 130, no. 7, pp. 578581, 1999.

[50] S. A. Wilbert, "Effect of passive smoking on angina pectoris," New England Journal of Medicine, vol. 299, no. 1, pp. 21-24, 1978.

[51] M. Kato, P. Roberts-Thomson, B. G. Phillips et al., "The effects of short-term passive smoke exposure on endotheliumdependent and independent vasodilation," Journal of Hypertension, vol. 17, no. 10, pp. 1395-1401, 1999.

[52] K. Ball and R. Turner, "Smoking and the heart. The basis for action," Lancet, vol. 2, no. 7884, pp. 822-826, 1974.

[53] P. Hill and E. L. Wynder, "Smoking and cardiovascular disease. Effect of nicotine on the serum epinephrine and corticoids," American Heart Journal, vol. 87, no. 4, pp. 491-496, 1974.

[54] P. E. Cryer, M. W. Haymond, J. V. Santiago, and S. D. Shah, "Norepinephrine and epinephrine release and adrenergic mediation of smoking associated hemodynamic and metabolic events," New England Journal of Medicine, vol. 295, no. 11, pp. 573-577, 1976.

[55] S. M. Ayres, H. S. Mueller, J. J. Gregory, S. Giannelli, and J. L. Penny, "Systemic and myocardial hemodynamic responses to relatively small concentrations of carboxyhemoglobin (COHB)," Archives of Environmental Health, vol. 18, no. 4, pp. 699-709, 1969.

[56] A. Mahmud and J. Feely, "Effects of passive smoking on blood pressure and aortic pressure waveform in healthy young adults-influence of gender," British Journal of Clinical Pharmacology, vol. 57, no. 1, pp. 37-43, 2004.

[57] A. Leone and F. Corsini, "Carboxyhaemoglobin from passive smoking more than age influences blood pressure," American Journal of Hypertension, vol. 14, p. 242, 2001.

[58] M. A. Samuels, "Neurally induced cardiac damage: definition of the problem," Neurologic Clinics, vol. 11, no. 2, pp. 273-292, 1993.

[59] J. Barnoja and S. A. Glantz, "Cardiovascular effects of secondhand smoke: nearly as large as smoking," Circulation, vol. 111, no. 20, pp. 2684-2698, 2005.

[60] D. L. Cohen and R. R. Townsend, "Does cigarette use modify blood pressure measurement or the effectiveness of blood 
pressure medications?" Journal of Clinical Hypertension, vol. 11, no. 11, pp. 657-658, 2009.

[61] F. R. Buhler, K. Vesanen, J. T. Watters, and P. Bolli, "Impact of smoking on heart attacks, strokes, blood pressure control, drug dose, and quality of life aspects in the International Prospective Primary Prevention Study in Hypertension," American Heart Journal, vol. 115, no. 1, pp. 282-288, 1988.

[62] P. Bolli, F. R. Buhler, and J. K. McKenzie, "Smoking, antihypertensive treatment benefit, and comprehensive antihypertensive treatment approach: some thoughts on the results of the international prospective primary prevention study in hypertension," Journal of Cardiovascular Pharmacology, vol. 16, no. 7, pp. S77-S80, 1990.

[63] H. E. F. Davies, "Respiratory changes in heart rate, sinus arrhythmias in the elderly," Gerontologia Clinica, vol. 17, pp. 96-100, 1975.

[64] B. Gribbin, T. G. Pickering, P. Sleight, and R. Peto, "Effect of age and high blood pressure on baroreflex sensitivity in man," Circulation Research, vol. 29, no. 4, pp. 424-431, 1971.

[65] D. A. Rothbaum, D. J. Shaw, C. S. Angell, and N. W. Shock, "Cardiac performance in the unanesthetized senescent male rat," Journals of Gerontology, vol. 28, no. 3, pp. 287-292, 1973.

[66] R. E. Vestal, A. J. J. Wood, and D. G. Shand, "Reduced $\beta$ adrenoceptor sensitivity in the elderly," Clinical Pharmacology and Therapeutics, vol. 26, no. 2, pp. 181-186, 1979.

[67] M. L. Weisfeldt, "Aging of the cardiovascular system," New England Journal of Medicine, vol. 303, no. 20, pp. 1172-1174, 1980.

[68] A. C. Schmidt, B. Flick, E. Jahn, and P. Bramlage, "Effects of the vasodilating beta-blocker nebivolol on smoking-induced endothelial dysfunction in young healthy volunteers," Vascular Health and Risk Management, vol. 4, no. 4, pp. 909-915, 2008.

[69] L. J. Scott, J. H. Warram, L. S. Hanna, L. M. B. Laffel, L. Ryan, and A. S. Krolewski, "A nonlinear effect of hyperglycemia and current cigarette smoking are major determinants of the onset of microalbuminuria in type 1 diabetes," Diabetes, vol. 50, no. 12, pp. 2842-2849, 2001.

[70] M. D. Winniford, D. E. Jansen, and G. A. Reynolds, "Cigarette smoking-induced coronary vasoconstriction in atherosclerotic coronary artery disease and prevention by calcium antagonists and nitroglycerin," American Journal of Cardiology, vol. 59, no. 4, pp. 203-207, 1987.

[71] B. Beermann and M. Groschinsky-Grind, "Clinical pharmacokinetics of diuretics," Clinical Pharmacokinetics, vol. 5, no. 3, pp. 221-245, 1980.

[72] A. Lant, "Diuretics: clinical pharmacology and therapeutic use," Drugs, vol. 29, no. 1, pp. 57-87, 1985.

[73] C. T. Dollery, M. Harington, and G. Kaufmann, "The mode of action of chlorothiazide in hypertension: with special reference to potentiation of ganglion-blocking drugs," The Lancet, vol. 273, no. 7085, pp. 1215-1218, 1959.

[74] J. H. Burn, L. H. Truelove, and I. Burn, "Antidiuretic action of nicotine and of smoking," British Medical Journal, vol. 1, pp. 403-406, 1945.

[75] H. Iida, M. Iida, M. Takenaka, H. Fujiwara, and S. Dohi, "Angiotensin II type 1 (AT1)-receptor blocker prevents impairment of endothelium-dependent cerebral vasodilation by acute cigarette smoking in rats," Life Sciences, vol. 78, no. 12, pp. 1310-1316, 2006.

[76] E. Beall, “The contents of Hesiod's Pandora Jar," Hermes, vol. 117, pp. 227-230, 1989.

[77] T. K. Makris, C. Thomopoulos, D. P. Papadopoulos et al., "Association of passive smoking with masked hypertension in clinically normotensive nonsmokers," American Journal of Hypertension, vol. 22, no. 8, pp. 853-859, 2009.

[78] A. Leone and A. Balbarini, "Tobacco smoke: a friend-enemy of blood pressure," Journal of Clinical Hypertension, vol. 12, supplement 1, p. 123, 2010. 


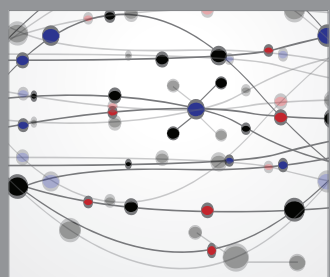

The Scientific World Journal
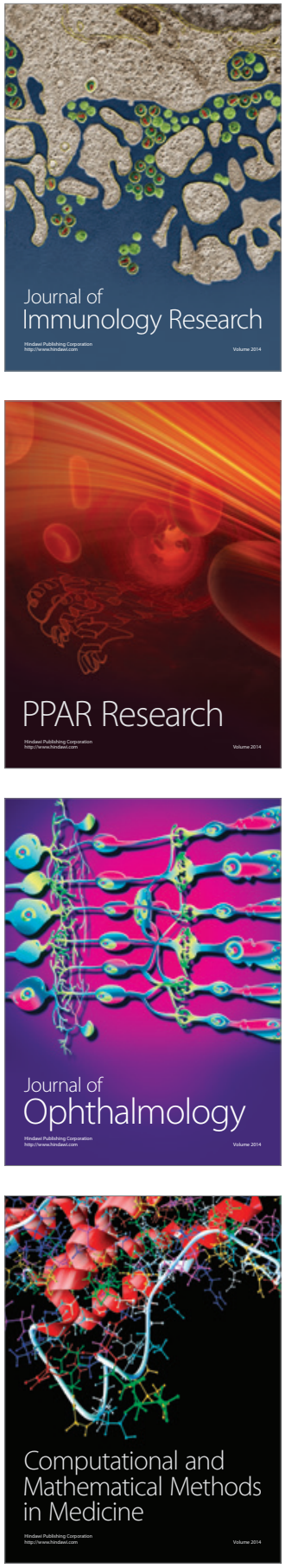

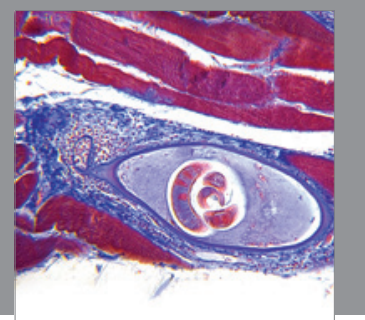

Gastroenterology

Research and Practice
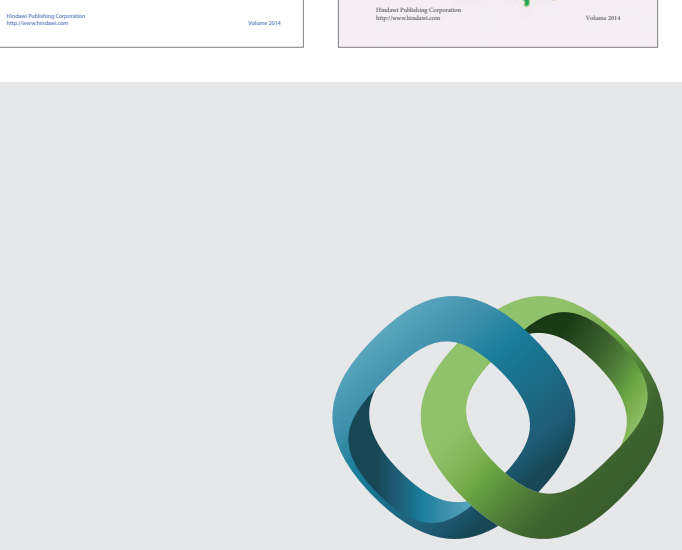

\section{Hindawi}

Submit your manuscripts at

http://www.hindawi.com
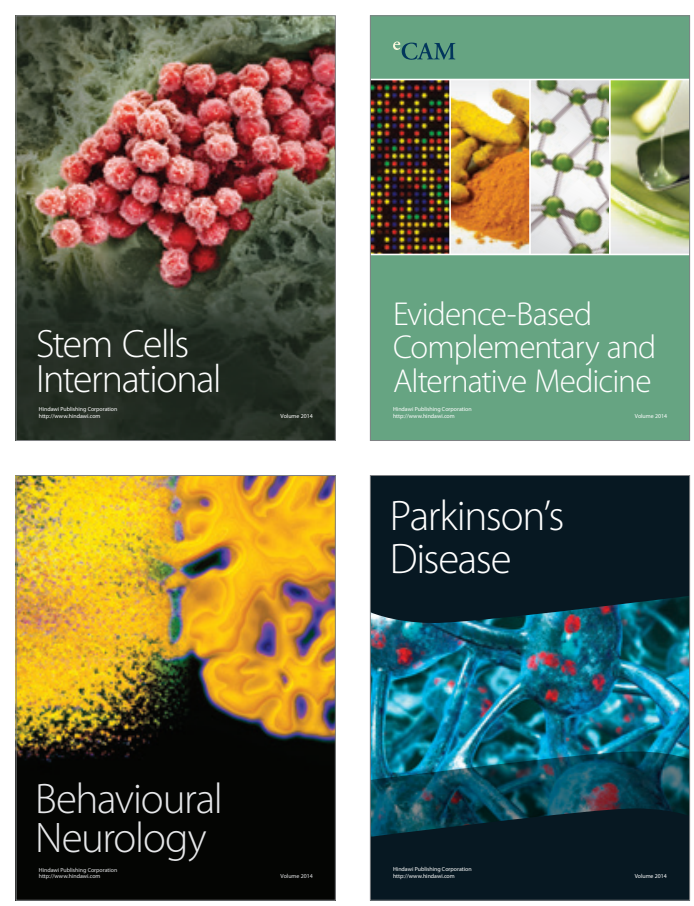

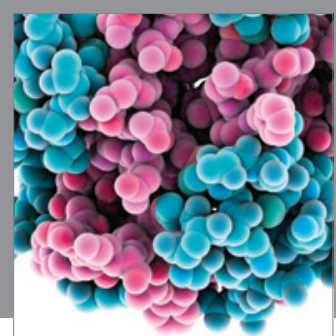

Journal of
Diabetes Research

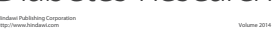

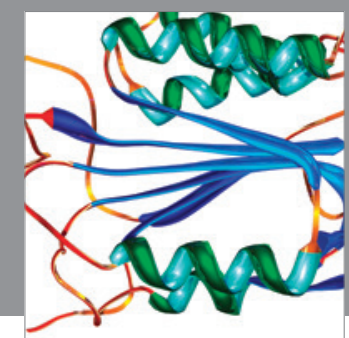

Disease Markers
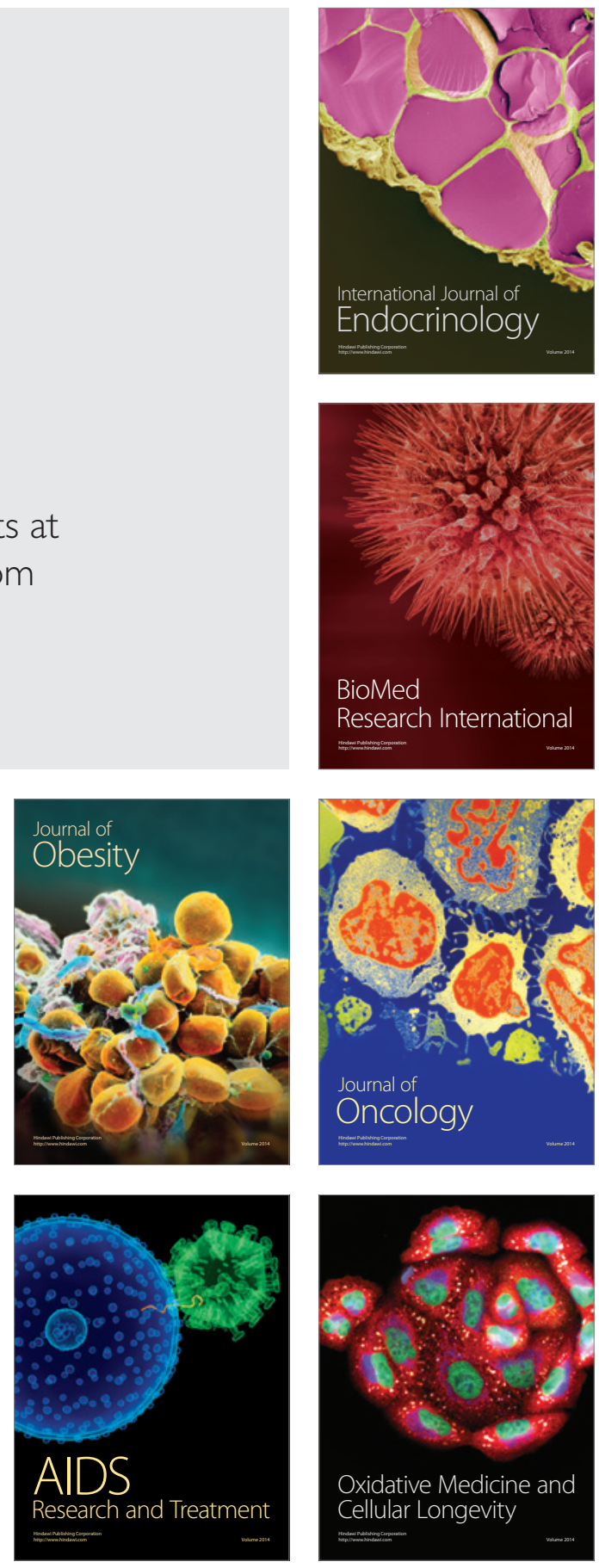